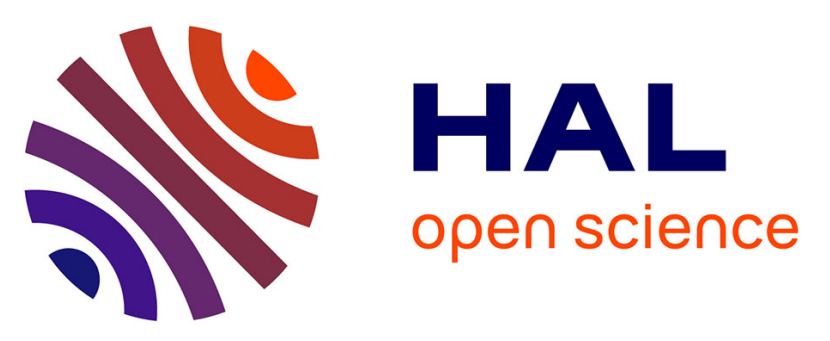

\title{
Numerical and experimental analysis of nonlinear regenerative amplifiers overcoming the gain bandwidth limitation
}

Julien Pouysegur, Martin Delaigue, C. Honninger, Yoann Zaouter, Patrick Georges, Frédéric Druon, Eric Mottay

\section{To cite this version:}

Julien Pouysegur, Martin Delaigue, C. Honninger, Yoann Zaouter, Patrick Georges, et al.. Numerical and experimental analysis of nonlinear regenerative amplifiers overcoming the gain bandwidth limitation. IEEE Journal of Selected Topics in Quantum Electronics, 2015, 21 (1), pp.1600208. 10.1109/JSTQE.2014.2321520 . hal-01222036

\section{HAL Id: hal-01222036 \\ https://hal.science/hal-01222036}

Submitted on 29 Oct 2015

HAL is a multi-disciplinary open access archive for the deposit and dissemination of scientific research documents, whether they are published or not. The documents may come from teaching and research institutions in France or abroad, or from public or private research centers.
L'archive ouverte pluridisciplinaire HAL, est destinée au dépôt et à la diffusion de documents scientifiques de niveau recherche, publiés ou non, émanant des établissements d'enseignement et de recherche français ou étrangers, des laboratoires publics ou privés. 


\title{
Numerical and experimental analysis of nonlinear regenerative amplifiers overcoming the gain bandwidth limitation
}

\author{
Julien POUYSEGUR, Martin DELAIGUE, Clemens HÖNNINGER, Yoann ZAOUTER, Patrick \\ GEORGES, Frédéric DRUON, Eric MOTTAY
}

\begin{abstract}
We present a numerical and experimental analysis of a nonlinear architecture to overcome the gain bandwidth limitation in regenerative amplifiers. This technique is based on the optimization of dispersion and nonlinear effects during the amplification process to obtain broad-bandwidth pulses that can be compressed to short durations with high temporal quality. We demonstrate the advantage of this method to maintain an excellent temporal quality of pulses even at high levels of optical nonlinearity. The technique has been applied to regenerative amplifiers using Yb:YAG, Yb:KYW, and Yb:CALGO crystals as gain media. In all cases we achieved the shortest pulse duration ever obtained from regenerative amplifiers using the respective laser crystals. These results underline the benefits of this amplification technique with respect to current state of the art.
\end{abstract}

Index Terms - Regenerative Amplifiers, Ultrafast laser, Nonlinear optics, Diode-pumped solid state laser

\section{INTRODUCTION}

$\mathrm{N}$ OWADAYS, femtosecond diode-pumped Yb-doped solid-state laser (fs DPSSL) systems are rapidly entering industrial processing because of their unique properties allowing machining with unprecedented quality and precision [1-2]. The diode pumping technology applied to Yb-dopedmaterials allows compact, robust and efficient femtosecond laser systems. However, some emerging industrial applications, in particular processing of dielectric or semiconductor materials where the laser-matter interaction relies on multi-photon processes, would benefit from short pulse duration of less than $300 \mathrm{fs}$ with few tens to few hundreds of $\mu \mathrm{J}$ of energy per pulse. These requirements have proven to be quite challenging for $\mathrm{Yb}$-doped gain matrices that do not exhibit sufficiently large gain bandwidth to provide high optical gain for such short pulses. In particular, this statement holds true for the workhorse Yb-doped crystals that are $\mathrm{Yb}: \mathrm{YAG}$ and $\mathrm{Yb}$ :Tungstate which typically generate pulses in the 400 fs to 1 ps pulse duration after regenerative amplification.

Manuscript received XXXX

J. Pouysegur, M. Delaigue, C. Hönninger Y. Zaouter, and E. Mottay are with Amplitude Systèmes, 11 avenue de Canteranne, Cité de la Photonique, 33600 Pessac, France (email: jpouysegur@amplitude-systemes.com).

F. Druon and P. Georges are with the Laboratoire Charles Fabry, Institut d'Optique, CNRS, Université Paris Sud, 91127 Palaiseau, France
In chirped pulse amplifiers (CPA) [3], the minimum obtainable pulse duration is ultimately limited by gain narrowing. For a given gain factor $\mathrm{G}$, this gain narrowing impact can be simply evaluated, considering Gaussian spectral shapes, as given by [4]

$$
t_{\text {out }}=t_{\text {in }} \sqrt{1+4 \ln (2) G /\left(\pi^{2} \Delta v^{2} t_{\text {in }}{ }^{2}\right)},
$$

where $t_{\text {in }}$ and $t_{\text {out }}$ are input and compressed output full width at half maximum (FWHM) pulse durations, and $\Delta v$ is the spectral bandwidth of the gain medium.

A large value of $G$, which is typical in regenerative amplifiers, leads to a significant reduction of the spectral bandwidth and consequently an increase of amplified pulse duration. Of course, this effect can be partially mitigated by selecting an $\mathrm{Yb}$-doped matrix with a large gain bandwidth $\Delta \boldsymbol{v}$. Due to its high crystalline order, Yb:YAG exhibits a relatively narrow spectral gain bandwidth despite having excellent thermal properties (with a thermal conductivity around 10 $\mathrm{W} / \mathrm{m} / \mathrm{K}$ for an undoped crystal) and one of the highest emission cross-section among Yb-doped materials. Due to these properties, $\mathrm{Yb}: \mathrm{YAG}$ regenerative amplifiers can generate high average power and high energy pulses, at the expense of long (ps) pulse durations due to the very high gain involved, typically $>40 \mathrm{~dB}$ [5-6]. Yb:Tungstate materials such as $\mathrm{Yb}: \mathrm{KYW}$ offer a valid alternative for generating shorter pulses at high gain levels. Indeed, the gain bandwidth of this Yb-doped matrix is broader than Yb:YAG while crosssections remain high and thermal conductivity acceptable (around $3 \mathrm{~W} / \mathrm{m} . \mathrm{K}$ ). Therefore, with $\mathrm{Yb}: \mathrm{KYW}$, high energy pulses with medium level of average power (few watts to few tens of watts) can be achieved with a pulse duration limited by gain narrowing to the $300-500$ fs range [7-8].

To generate even shorter pulses, broader gain bandwidth media with very disordered matrices [9-10], or a combination of gain media [11-13] can be used. The latter can be done either in series, where the beam to be amplified propagates successively in gain media with slightly shifted gain bandwidth, or in parallel using recently demonstrated femtosecond spectral coherent combining architectures [1415]. These strategies can lead to pulses shorter than $200 \mathrm{fs}$ but are rather complex, which currently prevents their use outside an optical laboratory. 
A second architecture consists in shortening amplified pulses using well-known temporal post-compression techniques [16-17], whereby spectral content is generated by self-phase modulation (SPM) in a guiding or non-guiding nonlinear medium. Subsequent dispersive elements allow a significant temporal compression. Although many interesting demonstrations have been done using this approach, its complexity and sensitivity to alignment still prevent it from spreading outside scientific laboratories.

Finally, SPM accumulated during amplification can be used to broaden the spectrum and compensate for gain narrowing. This approach has been widely studied in the context of fiber amplification and also provided promising results in the context of solid-state regenerative amplification. In particular, removal of the stretcher, typically used in CPA configuration, and therefore direct seeding of ultrashort pulses in the regenerative amplifier leads through nonlinear amplification to the generation of 250 to 350 fs pulses from $\mathrm{Yb}: \mathrm{KYW}$ and $\mathrm{Yb}: \mathrm{YAG}$, respectively [18-19] with moderate to poor temporal quality [20].

In this article, we introduce a novel strategy for the generation of ultrashort pulses with duration well below the conventional gain bandwidth-limited value by optimizing the chirp of the seed pulse prior to injecting it into the regenerative amplifier cavity. The carefully chosen initial chirp is fundamental in this technique and leads to a specific amplification behavior as the pulse accumulates roundtrips in the regenerative amplifier. This regime allows the generation of very short pulses together with an unprecedented temporal quality after compression. In section II we present in detail the concept of this nonlinear amplification. Section III provides a theoretical analysis for different gain bandwidths and initial chirps in order to highlight the unique dynamics of this nonlinear amplification technique. Finally, in section IV, we present some recent results demonstrating high temporal quality ultrashort and energetic pulse generation beyond the gain narrowing-limited compression duration based on this innovative scheme with $\mathrm{Yb}: \mathrm{YAG}, \mathrm{Yb}: \mathrm{KYW}$ and $\mathrm{Yb}$ :CALGO gain media.

\section{AMPLIFICATION PRINCIPLE}

Unlike typical nonlinear amplifiers where either Fourier transform-limited pulses or pulses with a moderate positive chirp are seeded to the amplifier, our technique relies on the precise control of a negative initial chirp of the seed pulse. The typical layout of the system is shown in Figure 1.

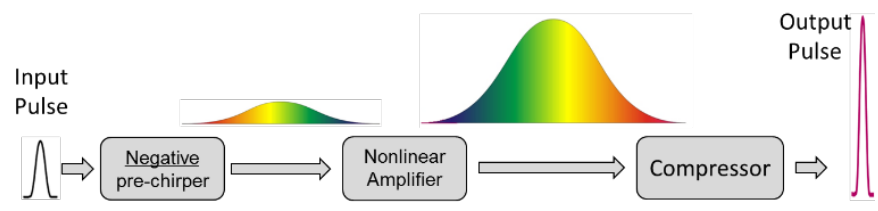

Fig 1: Experimental setup of the nonlinear regenerative amplifier.
The pulses are typically generated by an ultrafast oscillator at high repetition rate. Before amplification, a dispersive unit is inserted to control the initial amount of negative chirp before seeding to the regenerative amplifier. The negatively chirped pulses are then amplified to an energy level in the 10 $500 \mu \mathrm{J}$ range. In the regenerative amplifier, the mode sizes are carefully adjusted for the pulses to accumulate a significant amount of SPM while remaining below optical damage thresholds in the optical components such as the Pockels cell, polarizer, and gain medium. Finally, a compressor is used to reach the minimum pulse duration and reveal the maximum peak intensity.

The amplification behavior that is described in details in the following simulation section relies on a complex interplay between initial chirp, gain bandwidth, amount of gain, dispersion, and self-phase modulation as the dominant nonlinear effect. Because of this, the optimal operation point to obtain ultrashort and high temporal quality pulses relies on three consecutive amplification steps.

In the first stage of amplification, positive dispersion due to the propagation through the optical components in the amplifier cavity compensates for the initial negative chirp until the peak intensity becomes high enough to generate a substantial amount of SPM. This is the turning point for the second amplification step. At this point, the remaining chirp is still negative. Therefore, SPM-induced spectral compression [21-22] occurs and severely narrows the spectral content of the pulses until the remaining chirp is completely compensated. Then, in the third step, SPM together with positive dispersion broadens the spectrum quickly in the last few roundtrips of amplification.

The SPM-induced spectral compression stage is essential and acts as a spectral phase cleaner before the large spectral broadening occurs, leading to high temporal quality pulses after compression. We show that any substantial deviation from the parameters that leads to this three-step evolution results in either longer and/or temporally degraded compressed pulses.

\section{NUMERICAL SIMULATIONS}

In order to highlight the benefits of optimizing the initial negative chirp in nonlinear amplification, we perform simulations for different cases. First, a hypothetical broadband material is studied as a reference. Indeed, the processes involved in nonlinear amplification such as gain-narrowing, spectral compression and spectral broadening, can be clearly identified, and their roles are emphasized. In particular, we show the strong influence of the exact value of the initial negative chirp on the output pulse duration and temporal quality. Second, we use parameters of a $\mathrm{Yb}: \mathrm{KYW}$ crystal, and compare the amplification dynamics in two cases: Fouriertransform limited seed and negatively chirped seed. The framework of the simulations corresponds to the experimental goal of reaching the $30 \mu \mathrm{J}$ range with a gain of $10^{5}-10^{6}$ in the amplifier while achieving short output pulse duration and preserving a good temporal quality. 
Simulations are based on a split-step Fourier algorithm to solve the nonlinear Schrödinger propagation equation. The pulse evolution in energy, duration and spectrum is examined along the amplification process. The modeled elements include the Pockels cell, laser crystal, and polarizer. Only Group Velocity Dispersion (GVD) and Third Order Dispersion (TOD) are considered in the simulations.

\section{A. Broadband material}

The parameters used for these simulations correspond to a broadband medium with $60 \mathrm{~nm}$ FWHM emission spectrum amplifying pulses from $100 \mathrm{pJ}$ to $30 \mu \mathrm{J}$, i. e. with a $54 \mathrm{~dB}$ gain. Figure 2 shows the evolution of pulse duration and spectral bandwidth during amplification in the case of an optimized input negative chirp. In this case the induced nonlinearity corresponds to a B-integral of $17 \mathrm{rad}$.
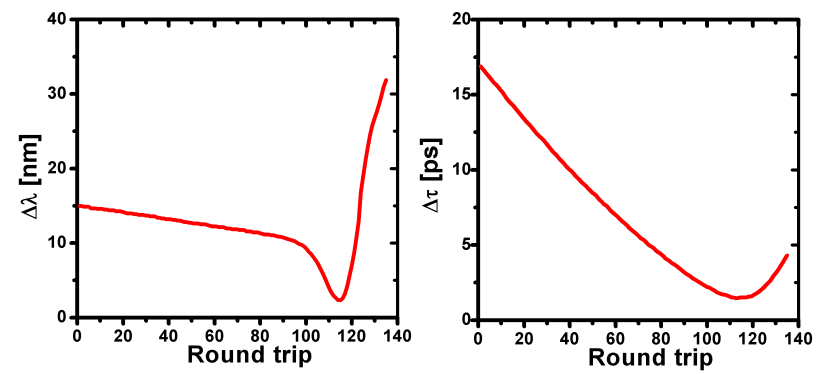

Fig. 2: Evolution of the pulse duration for an optimized precompensation of the dispersion (Left) during amplification. Evolution of the corresponding spectral bandwidth (Right)

This simulation can be considered as a reference and the three above-mentioned steps are clearly identified, especially in the pulse spectrum evolution (fig. 2 right). First, between roundtrips 1 and 100, gain narrowing in linear amplification regime induces a slow decrease of the bandwidth. Second, between round-trips 100 and 115 , the spectral bandwidth collapses due to SPM-induced spectral compression. Third, between roundtrips 115 and 140 a quick spectral broadening occurs due to SPM in the positive dispersion regime. In the time domain, the pulse width decreases during the two first steps and increases in the final spectral broadening step where a large nonlinear phase is accumulated. In this optimized case, the final spectral bandwidth of $>30 \mathrm{~nm}$ exceeds by a factor of two the initial 15 $\mathrm{nm}$ input bandwidth and therefore can support shorter pulses. In order to determine how critical the amount of initial negative chirp is to obtain short output pulses with good temporal quality, we now compare this optimal case to two situations where the initial negative chirp is either larger or smaller than the optimal one in absolute value.
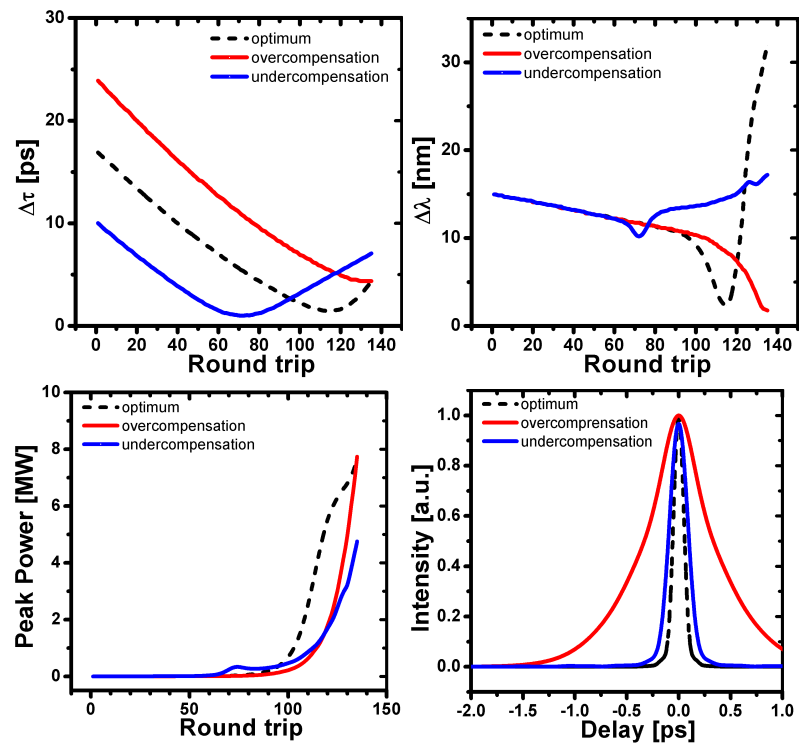

Fig 3: Top: Pulse duration (Left) and spectral bandwidth (Right) as a function of roundtrip number - Bottom (Left): peak power as a function of roundtrip number - (Right) Autocorrelation trace of compressed pulses. Except for the input chirp the parameters are the same as in the simulation of fig. 2. Indicated are the cases for optimum pre-chirp as well as for a too large negative pre-chirp (overcompensation) and a too weak negative pre-chirp (undercompensation).

We first focus on an intuitive case where the pre-chirp exactly compensates for the positive dispersion accumulated during the amplifier roundtrips. This chirp is larger in absolute value than the optimal one, and is further denoted as overcompensated. The pulse duration keeps decreasing during the entire amplification process and only approaches zero net chirp at the very end of the amplification process. Self-phase modulation therefore leads to spectral compression during the last few amplification roundtrips and no further spectral broadening is observed. This can be clearly observed in fig. 3 red curves) where the spectrum first decreases due to gain narrowing and finally collapses due to nonlinear spectral compression. The output spectrum only exhibits $2 \mathrm{~nm}$ bandwidth compared to the $15 \mathrm{~nm}$ spectrum width of the input pulses. At the output of the amplifier, the peak power is comparable to the optimal case but due to the narrow spectrum and the negligible remaining spectral phase, the pulse duration after compression is long (Fig. 3 - red curves). This overcompensated chirp situation is clearly not optimal and leads to performances that are below those of a purely linear amplifier limited by gain narrowing in terms of pulse duration.

Inversely, if we now consider the case where the input negative chirp is smaller in absolute value (undercompensated case) than the optimal case, the minimum pulse duration occurs too early in the amplification process. This leads to an early onset of self-phase modulation, but the positive dispersion accumulated thereafter temporally broadens the pulse and leads to a narrower final spectral bandwidth compared to the optimal case (Fig. 3 - blue curves). After compression, the pulse is longer than in the optimal case. 
These simulations indicate that in the case of a broadband material, nonlinear spectral compression dominates over gain narrowing only for a well-adjusted initial negative chirp (Fig. 3 - dashed curves). As we will see in the following, in the case of narrower bandwidth materials such as $\mathrm{Yb}: \mathrm{KYW}$ or $\mathrm{Yb}: \mathrm{YAG}$, the role of the negative pre-chirp is more subtle.

\section{B. $Y b: K Y W$ as an example of a narrower bandwidth material}

The role of optimal pre-chirp in nonlinear amplification is clearly identified in the previous section. We now examine the case of standard available Yb-doped materials that exhibit smaller gain bandwidths. In particular, we address two questions. First, in these gain media showing narrower bandwidth, is the transition between gain narrowing and nonlinear pulse compression so clear and important? Second, may the reduced amount of spectral broadening in the undercompensated case be balanced by experimentally increasing the nonlinearity in the amplifier? Simulations are carried out considering an Yb:KYW with $20 \mathrm{~nm}$ FWHM gain spectrum regenerative amplifier. The gain of the amplifier is $54 \mathrm{~dB}$.

We first compare the optimal pre-chirp case and, as an extreme undercompensated case, a Fourier transform-limited input pulse. The spot sizes and gains are assumed to be the same in both cases. Fig. 4 shows the results for an optimum negative pre-chirp (red), and for a Fourier transform-limited seed pulse (blue). The amplifier using pre-chirp still shows a significant advantage in terms of output pulse width and temporal quality, although the spectral compression transition is much less pronounced.
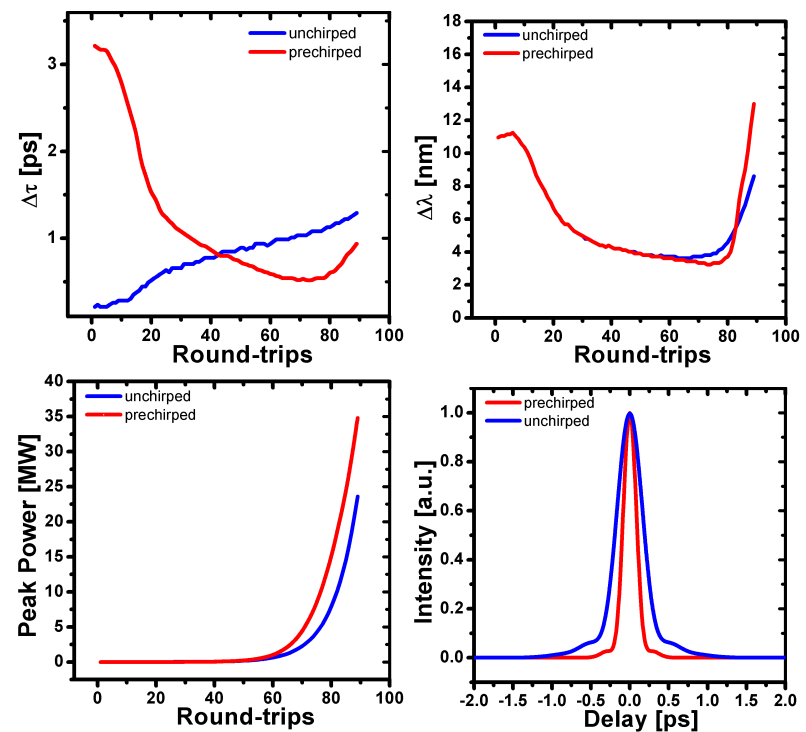

Fig.4. Top: Pulse duration (Left) and spectral bandwidth (Right) as a function of roundtrip number - Bottom (Left): peak power as a function of roundtrip number - (Right) Autocorrelation trace of compressed pulses. Red: optimal prechirped seed. Blue: unchirped seed.

The pre-chirped seed case shows a larger spectral bandwidth at the output, leading to the conclusion that the unchirped seed case might show better performances if the nonlinearity level is increased by changing the spots sizes in the amplifier elements. We therefore carry out simulations where the only changed parameter is the nonlinearity level in the unchirped seed case, in order to match the output spectral bandwidth to that of the pre-chirped seed case. The results are plotted in fig. 5.
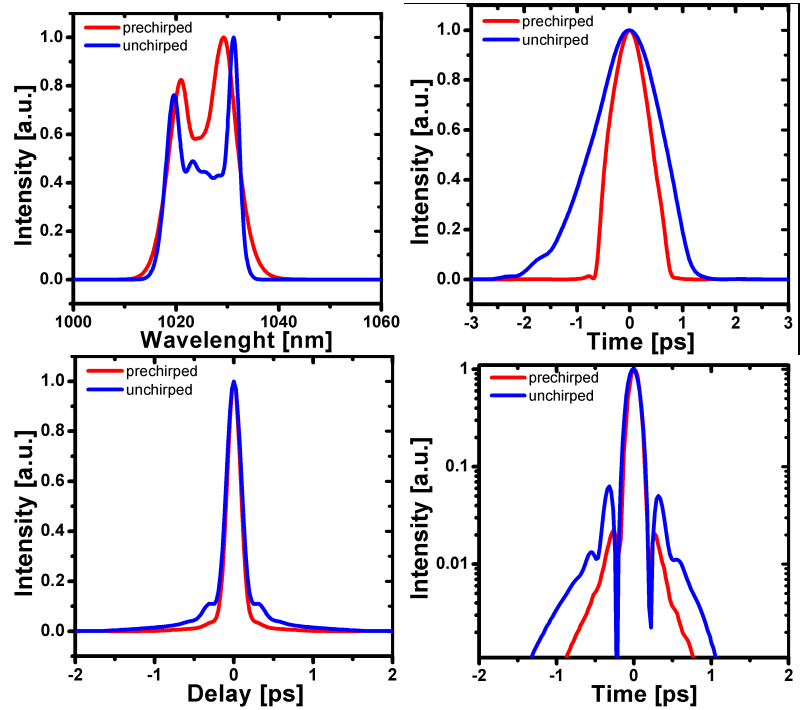

Fig. 5: Top: Output spectra (Left) - Temporal intensities of amplified pulses before compression (Right) - Bottom: Autocorrelation trace of compressed pulses (Left) - Temporal intensities of compressed pulses in logarithmic scale (Right). Red: optimal pre-chirped seed. Blue: unchirped seed.

In both cases the accumulation of nonlinear effects is well controlled and leads to a good compression close to the Fourier transform limit, with a temporal Strehl ratio in the 0.94-0.96 range (Fig.5-Bottom-Left). However, stronger pedestals appear compared to pre-chirped case. The value of the B-integral in the unchirped seed case is $6 \mathrm{rad}$, while it is 7 rad for the optimal pre-chirped case. However, in the prechirped case, spectral compression leads to a quasi-Fourier transform limited pulse close to the end of the amplification process, at roundtrip number 80 . The $\mathrm{B}$ integral accumulated from this point is reduced to $5.9 \mathrm{rad}$. This also allows a more symmetric temporal shape of the uncompressed pulse at the output, together with a smoother spectral shape that reduces the amount of energy in the temporal pedestal (see Fig 5-TopRight). To quantify the pulse quality in both cases, we calculate the amount of energy contained in the main pulse, assuming a Gaussian shape for the integral-overlap. For the pre-chirped seed pulse this value is $96 \%$ while it is reduced to $87 \%$ for the unchirped seed pulse. These simulations confirm the advantage of an optimized negative input chirp for nonlinear amplification in terms of output pulse width and temporal quality.

\section{EXPERIMENTAL RESULTS}

We now present experimental results that were all obtained using the nonlinear architecture described in fig. 1. We used this configuration with three different regenerative amplifiers. 
The first one is a high power system based on an Yb:YAG thin disk-crystal [23]; the second one is a short pulse system based on $\mathrm{Yb}: \mathrm{KYW}$, and the third one is an ultrashort pulse system based on a broadband Yb:CALGO crystal in order to investigate limitations in pulse duration.

\section{A. Yb:YAG Thin Disk regenerative amplifier}

The seed is a fiber oscillator delivering $\sim 10 \mathrm{~nm}$ bandwidth pulses at $41 \mathrm{MHz}$ at an average power of $10 \mathrm{~mW}$. An initial negative chirp is applied to the pulses and leads to a duration prior to amplification of $6 \mathrm{ps}$. The gain medium is a $220 \mu \mathrm{m}$ Yb:YAG thin-disk with $7 \%$ doping concentration. It is pumped with a $150 \mathrm{~W}$ laser diode at $940 \mathrm{~nm}$. The amplifier operates at $36 \mathrm{~W}$ average power. At $100 \mathrm{kHz}, 360 \mu \mathrm{J}$ pulses are generated, and the output autocorrelation trace FWHM is 400 fs (Fig. 6-Right). Assuming an autocorrelation deconvolution factor of 0.7 , this leads to an estimated pulse duration of $295 \mathrm{fs}$.

The estimated value of the B-integral in the system is $10 \mathrm{rad}$. This large amount of nonlinearity is visible in the measured spectrum; the spectrum has 3 lobes for an overall $10 \mathrm{~nm}$ FWHM bandwidth (Fig 6-Left).
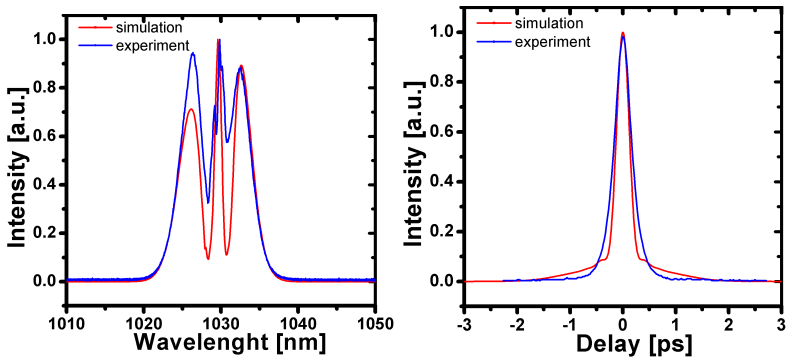

Fig 6: Experimental (blue) and simulated (red) spectra (Left) and autocorrelations (Right) at the output of the Yb:YAG thin disk regenerative amplifier.

Compression of the pulses in performed using a set of dispersive Gires-Tournois-Interferometer (GTI)-based mirrors, leading to a compressor transmission greater than $95 \%$. However, a slight difference between simulated and measured autocorrelation is observed, that we attribute to imperfect dispersion compensation by this set of GTI. Still, the achieved duration is significantly shorter than what is typically obtained with such gain medium, with a pedestal-free autocorrelation. The output pulse energy is $>300 \mu \mathrm{J}$ with $<300$ fs pulse for a gain of $60 \mathrm{~dB}$. These performances are unprecedented in terms of pulse duration for a high gain Yb:YAG amplifier.

To decrease the pulse duration even further, we applied this technique to a regenerative amplifier based on $\mathrm{Yb}: \mathrm{KYW}$.

\section{B. $Y b: K Y W$ regenerative amplifier}

The laser oscillator is almost identical to the one used for the $\mathrm{Yb}: Y A G$ amplifier and delivers pulses centered at $1030 \mathrm{~nm}$ with a spectral bandwidth of $\sim 10 \mathrm{~nm}$ at $40 \mathrm{MHz}$ and an average power of $10 \mathrm{~mW}$. The pulses are negatively prechirped leading to a moderately stretched pulse duration of less than $5 \mathrm{ps}$. The crystal used inside the amplifier is a $7 \mathrm{at} \%$ doped $\mathrm{Yb}: \mathrm{KYW}$ with $0.5 \mathrm{~mm}$ gain length. It is longitudinally pumped with a $15-\mathrm{W}, 400-\mu \mathrm{m}$-diameter-fiber-coupled laser diode emitting at $976 \mathrm{~nm}$. The pump beam is 1:1 imaged in the crystal. The cavity length is $3 \mathrm{~m}$ (corresponding to a round trip time of $20 \mathrm{~ns}$ ). In order to easily optimize the pulse duration, the compressor uses transmission gratings of 500 grooves per $\mathrm{mm}$ and exhibits an overall efficiency of $75 \%$.

At $50 \mathrm{kHz}$, the $\mathrm{Yb}: \mathrm{KYW}$ amplifier generates an energy of $32 \mu \mathrm{J}$ after compression with a pulse duration of $150 \mathrm{fs}$ and $1.6 \mathrm{~W}$ of average power, corresponding to a B-integral of 6 rad. The spectrum shows two lobes and exhibits a FWHM bandwidth of $14 \mathrm{~nm}$ (Fig. 7-Left). The FWHM duration of the autocorrelation is 203 fs. Numerical simulations have been performed and both the spectrum and the autocorrelation show a quantitative agreement as shown in Fig. 8. By this mean we can estimate that the temporal Strehl ratio is $96 \%$ with a peak power of $220 \mathrm{MW}$, and a pulse width of $150 \mathrm{fs}$ (Fig. 7-Right).
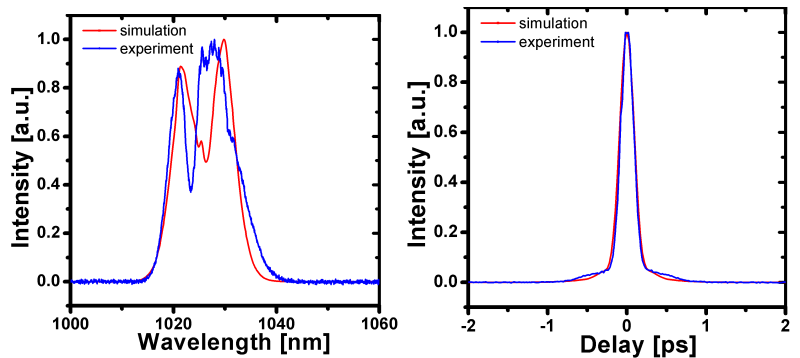

Fig 7: Experimental (blue) and simulated (red) spectra (Left) and autocorrelations (Right) at the output of the Yb:KYW thin disk regenerative amplifier.

Yb:KYW regenerative amplifier systems can therefore be substantially improved in terms of pulse duration by implementing this technique of nonlinear amplification. To decrease the amplified pulse width even more, we now turn to a less conventional broadband laser crystal.

\section{Yb:CALGO regenerative amplifier}

The Yb:CALGO material stands out among novel gain media because it has the broadest emission cross-sections together with good thermal and mechanical properties [24-25]. Indeed, with a emission bandwidth of $\sim 60 \mathrm{~nm}$ spanning from $1010 \mathrm{~nm}$ to $1070 \mathrm{~nm}$ and its thermal conductivity of 9.7 W.K ${ }^{-1}$ (undoped matrix), Yb:CALGO holds great promises with respect to average power scaling. Very encouraging results have already been demonstrated with this crystal in oscillator and linear CPA systems [26-30]. To decrease further the pulse duration in amplified systems, we have applied the nonlinear regenerative amplifier technique to this crystalline laser medium.

The ultrafast oscillator generates a train of 165 fs pulses centered at $1047 \mathrm{~nm}$ with a spectral bandwidth of $15 \mathrm{~nm}$ at 46 $\mathrm{MHz}$ and an average power of $10 \mathrm{~mW}$. Before seeding into the regenerative amplifier, the pulses are negatively stretched to $\sim 20$ ps by use of a $800 \mathrm{l} / \mathrm{mm}$ transmission grating-based dispersive line. The gain medium is a 4-mm-long AR-coated $5 \% \mathrm{Yb}$-doped CALGO cut along the $a$ axis. It is pumped at $980 \mathrm{~nm}$ with a fiber-coupled unpolarized laser diode delivering an average power of $12 \mathrm{~W}$. The pump beam is $1: 1$ imaged in the crystal through a dichroic mirror. The cavity 
length is $1.3 \mathrm{~m}$. A $500 \mathrm{l} / \mathrm{mm}$ grating-based compressor is used to compress the output chirped pulses with an overall efficiency of $60 \%$. This low value is due to the low diffraction efficiency of the grating used and spectral losses in some components of the compressor.

At $50 \mathrm{kHz}$, the $\mathrm{Yb}$ :CALGO amplifier generates output pulse energy of $24 \mu \mathrm{J}$ after compression with a pulse duration of $97 \mathrm{fs}$ for an average power or 1.2W [31]. The B-integral in this configuration is $17 \mathrm{rad}$. The results of the SHG-FROG measurement is confirmed by cross-checking with independent autocorrelation and spectrum measurements as shown in Fig. 8 and 9. The retrieved temporal profile shows a FWHM duration of $97 \mathrm{fs}$, excellent temporal quality, and quantitative agreement with simulations. The spectrum has a FWHM bandwidth of $19 \mathrm{~nm}$ corresponding to a timebandwidth product of 0.5 , again in good agreement with the numerical model. The temporal Strehl ratio is in this case $84 \%$ and the peak power $218 \mathrm{MW}$. In this experiment, the Fouriertransform limited pulsewidth is $87 \mathrm{fs}$.
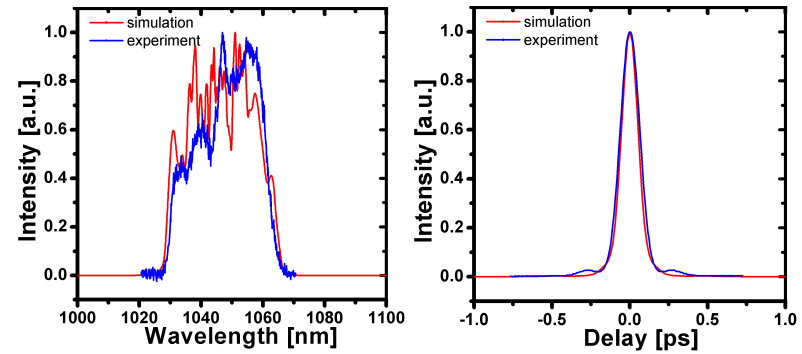

Fig 8: Experimental (blue) and simulated (red) spectra (Left) and autocorrelations (Right) at the output of the Yb:CALGO thin disk regenerative amplifier.
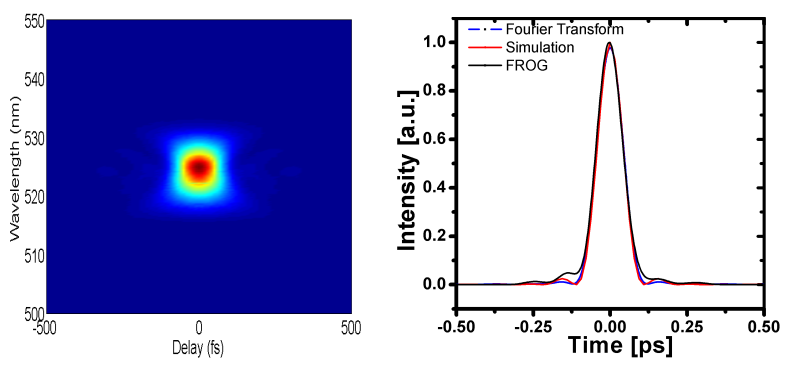

Fig 9: (Left) Measured FROG trace at $50 \mathrm{kHz}$ and temporal intensity retrieved from the FROG (black curve), simulation (red curve) and Fourier transform of the spectrum (blue curve)

Finally, we compare the evolution of the experimentally measured and simulated spectral bandwidths during amplification in order to validate our simulation and optimization approach, as shown in fig. 10. A quantitative agreement is observed, indicating that the model can be used to analyze the obtained results and to predict the behavior of the nonlinear regenerative amplification.

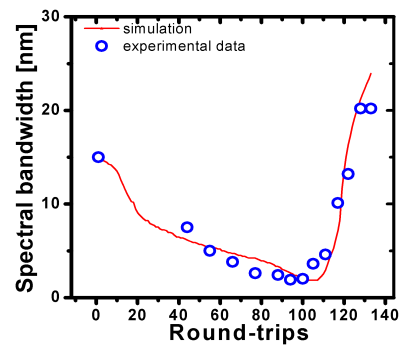

Fig 10: Experimental data and simulation of the spectral bandwidth evolution during amplification in an Yb:CALGO nonlinear regenerative amplifier.

\section{Synthesis}

Table 1 summarizes the parameters used in all numerical simulations. Table 2 summarizes the experimental data obtained with the three reported laser systems.

The main differences between the two first reported systems and the Yb:CALGO case are the following: first, the nonlinearities in the $\mathrm{Yb}$ :CALGO regenerative amplifier essentially originate from the gain medium, which is not the case in the other regenerative amplifiers due to the thin-disk geometry; second, the gain in Yb:CALGO is significantly lower which leads to more roundtrips for efficient energy extraction and hence a higher amount of negative dispersion for pre-compensation.

TABLE I

INPUT PARAMETERS

\begin{tabular}{||c|ccc||}
\hline & Yb:YAG & Yb:KYW & Yb:CALGO \\
\hline $\mathrm{n}_{2}\left(\mathrm{~m}^{2} / \mathrm{W}\right)$ & $6.9 \mathrm{e}-20$ & $8.7 \mathrm{e}-20$ & $1.15 \mathrm{e}-19$ \\
$\mathrm{n}_{2 \text {-pockels }}\left(\mathrm{m}^{2} / \mathrm{W}\right)$ & $6 \mathrm{e}-20$ & $6 \mathrm{e}-20$ & $6 \mathrm{e}-20$ \\
$\lambda(\mathrm{nm})$ & 1030 & 1030 & 1050 \\
$\omega_{\text {crystal }}(\mu \mathrm{m})$ & 800 & 200 & 100 \\
$\omega_{\text {pockels }}(\mu \mathrm{m})$ & 800 & 550 & 450 \\
$\mathrm{~L}_{\text {crystal }}(\mathrm{mm})$ & $8 \times 0.22$ & $4 \times 0.25$ & $2 \times 4$ \\
$\mathrm{~L}_{\text {pockels }}(\mathrm{mm})$ & 40 & 20 & 20 \\
$\beta^{(2)}{ }_{\text {crystal }}\left(\mathrm{ps}^{2} / \mathrm{m}\right)$ & 0.07 & 0.2 & 0.48 \\
$\beta^{(3)}{ }_{\text {crystal }}\left(\mathrm{ps}^{3} / \mathrm{m}\right)$ & $6.7 \mathrm{e}-5$ & $12.8 \mathrm{e}-5$ & $6 \mathrm{e}-5$ \\
$\beta^{(2)}{ }_{\text {pockels }}\left(\mathrm{ps}^{2} / \mathrm{m}\right)$ & 0.04 & 0.04 & 0.04 \\
$\beta^{(3)}{ }_{\text {pockels }}\left(\mathrm{ps}^{3} / \mathrm{m}\right)$ & $6.65 \mathrm{e}-5$ & $6.65 \mathrm{e}-5$ & $6.65 \mathrm{e}-5$ \\
\hline
\end{tabular}

TABLE II

OUTPUT PARAMETERS

\begin{tabular}{||l||ccc||}
\hline & Yb:YAG & Yb:KYW & Yb:CALGO \\
\hline$\Delta \tau_{\text {out }}(\mathrm{fs})$ & 300 & 150 & 97 \\
$\Delta \lambda(\mathrm{nm})$ & 10 & 14 & 19 \\
Power $(\mathrm{W})$ & 36 & 1.5 & 1.2 \\
Rep. rate $(\mathrm{kHz})$ & 100 & 50 & 50 \\
Energy $(\mu \mathrm{J})$ & 360 & 30 & 24 \\
Strehl ratio $(\%)$ & 75 & 96 & 84 \\
Peak power $(\mathrm{MW})$ & 1200 & 220 & 218 \\
\hline
\end{tabular}

In the general case, the saturation fluence may be an issue. Anyways, it appears in our case that this point is not critical 
for two reasons. Firstly, in all the described regenerative amplifiers, we are working with pulses fluences far below the saturation fluences of the different gain media. Secondly, the gain saturation only occurs in the few last roundtrips without major impact on the overall pulse evolution.

\section{CONCLUSION}

We analyze in detail, both, numerically and experimentally, a new nonlinear amplification technique optimized for regenerative amplifiers. Understanding the evolution of the pulse during the amplification process allows for optimizing the chirp of the input pulse in order to obtain the desired nonlinear effects, and ultimately the shortest possible output pulse duration at a given objective for the extracted pulse energy. We demonstrate the advantage of this method over direct seeding of unchirped pulses in the amplifier. In particular, the temporal quality of amplified pulses is shown to be significantly increased.

These results have been validated by experimental demonstrations using different gain media: sub-300 fs pulses with an energy of $360 \mu \mathrm{J}$ and a peak power exceeding $1 \mathrm{GW}$ are reported from an Yb:YAG-based amplifier. Amplified pulses as short as $150 \mathrm{fs}$ with an energy of $32 \mu \mathrm{J}$ are obtained from a regenerative amplifier based on $\mathrm{Yb}: \mathrm{KYW}$. Finally, we show that the use of a broadband crystal such as $\mathrm{Yb}: \mathrm{CaGdAlO}_{4}$ allows the generation of sub-100 fs pulse, thus bringing $\mathrm{Yb}$-doped materials amplifiers to a parameter regime so far reserved to more complex Ti:sapphire amplifiers. We believe this technique to be very promising for the development of compact industrial systems operating in the range from $10 \mathrm{~s}$ to $100 \mathrm{~s} \mu \mathrm{J}$.

\section{REFERENCES}

[1] P.P. Pronko, S.K. Dutta, D. Du, R.K. Singh, "Thermophysical effects in laser processing of materials with picosecond and femtosecond pulses", J. Appl. Phys. 78, 6233 (1995)

[2]C. Momma, B.N. Chichkov, S. Nolte, A. Tünnermann, "Short-pulse laser ablation of solid targets", Opt. Commun. 129, 134 (1996)

[3]Donna Strickland, Gerard Mourou, Optics Communications, Volume 55, Issue 6, 15, Pages 447-449 (1985).

[4] Siegman, Lasers, university Science Books, p. 359, equation (78))

[5]C. Hönninger, I. Johannsen, M. Moser, G. Zhang, A. Giesen, and U. Keller, "Diode-pumped thin-disk Yb:YAG regenerative amplifier," Applied Physics B, vol. 65, pp. 423-426, (1997).

[6]K. Sueda, S. Kawato, and T. Kobayashi, "LD pumped Yb:YAG regenerative amplifier for high average power short-pulse generation," Laser Physics Letters, vol. 5, no. 4, pp. 271-275, Apr. 2008.

[7]Hsiao-hua Liu, John Nees, and Gérard Mourou, "Directly diode-pumped $\mathrm{Yb}: \mathrm{KY}\left(\mathrm{WO}_{4}\right)_{2}$ regenerative amplifiers," Opt. Lett. 27, 722-724 (2002)

[8]A. Calendron, K. Wentsch, J. Meier, and M. J. Lederer, "High Power and High Energy Yb:KYW Regenerative Amplifier Using a Chirped Volume Bragg Grating," in Conference on Lasers and Electro-Optics/International Quantum Electronics Conference, OSA Technical Digest (CD) (Optical Society of America, 2009), paper CFD2.

[9] Sandrine Ricaud, Frédéric Druon, Dimitris N. Papadopoulos, Patrice Camy, Jean-Louis Doualan, Richard Moncorgé, Martin Delaigue, Yoann Zaouter, Antoine Courjaud, Patrick Georges, and Eric Mottay, "Short-pulse and high-repetition-rate diode-pumped $\mathrm{Yb}: \mathrm{CaF}_{2}$ regenerative amplifier," Opt. Lett. 35, 2415-2417 (2010)

[10] E. Caracciolo, M. Kemnitzer, A. Guandalini, F. Pirzio, J. Aus der Au, and A. Agnesi, "28-W, 217 fs solid-state $\mathrm{Yb}: \mathrm{CAlGdO}_{4}$ regenerative amplifiers," Opt. Lett. 38, 4131-4133 (2013)
[11] U. Buenting, H. Sayinc, and D. Wandt, "Regenerative thin disk amplifier with combined gain spectra producing $500 \mu \mathrm{J}$ sub 200 fs pulses," Optics Letters, vol. 17, no. 10, pp. 8046-8050, 2009.

[12] G. Kim, J. Yang, and D. Lee, "Femtosecond laser based on Yb: KYW crystals with suppression of spectral narrowing in a regenerative amplifier by spectral profiling of the pulse," Journal of Optical Technology, pp. 142-147, 2013.

[13] A. Buettner, U. Buenting, D. Wandt, J. Neumann, and D. Kracht, "Ultrafast double-slab regenerative amplifier with combined gain spectra and intracavity dispersion compensation," Opt. Express 18, 21973-21980 (2010)

[14] Nikolai B. Chichkov, Udo Bünting, Dieter Wandt, Uwe Morgner, Jörg Neumann, and Dietmar Kracht, "Spatially dispersive regenerative amplification of ultrashort laser pulses," Opt. Express 17, 24075-24083 (2009) [15] Florent Guichard, Marc Hanna, Laurent Lombard, Yoann Zaouter, Clemens Hönninger, Franck Morin, Frédéric Druon, Eric Mottay, and Patrick Georges, "Two-channel pulse synthesis to overcome gain narrowing in femtosecond fiber amplifiers," Opt. Lett. 38, 5430-5433 (2013)

[16] T. Damm, M. Kaschke, F. Noack, and B. Wilhelmi, "Compression of picosecond pulses from a solid-state laser using self-phase modulation in graded-index fibers," Opt. Lett. 10, 176-178 (1985)

[17] M. Nisoli, S. De Silvestri, O. Svelto, R. Szipöcs, K. Ferencz, Ch. Spielmann, S. Sartania, and F. Krausz, "Compression of high-energy laser pulses below 5 fs," Opt. Lett. 22, 522-524 (1997)

generation," Laser Physics Letters, vol. 5, no. 4, pp. 271-275, Apr. 2008. [18] Mikhail Larionov, Friedrich Dausinger, "50W thin disk laser with variable pulse duration," Proc. of SPIE, Vol. 7912

[19] Mikhail Larionov, Frank Butze, Detlef Nickel, and Adolf Giesen, "High-repetition-rate regenerative thin-disk amplifier with $116 \mu \mathrm{J}$ pulse energy and 250 fs pulse duration," Opt. Lett. 32, 494-496 (2007)

[20] G H Kim, J Yang, A V Kulik, E G Sall, S A Chizhov, V E Yashin and U Kang, "Power limitations and pulse distortions in an Yb: KGW chirpedpulse amplification laser system," Quantum Electronics. 43725 (2013) [21] Brian R. Washburn, John A. Buck, and Stephen E. Ralph, "Transformlimited spectral compression due to self-phase modulation in fibers," Opt. Lett. 25, 445-447 (2000)

[22] S. A. Planas, N. L. Pires Mansur, C. H. Brito Cruz, and H. L. Fragnito, "Spectral narrowing in the propagation of chirped pulses in single-mode fibers," Opt. Lett. 18, 699-701 (1993)

[23] A. Giesen, J. Speiser, "Fifteen Years of Work on Thin-Disk Lasers: Results and Scaling Laws," Quantum Electronics, IEEE. 13, 598-609 (2007)

[24] Johan Petit, Philippe Goldner, and Bruno Viana, "Laser emission with low quantum defect in Yb:CaGdAlO4," Opt. Lett. 30, 1345-1347 (2005)

[25] F. Druon, M. Olivier, A. Jaffrès, P. Loiseau, N. Aubry, J. DidierJean, F. Balembois, B. Viana, and P. Georges, "Magic mode switching in $\mathrm{Yb}: \mathrm{CaGdAlO}_{4}$ laser under high pump power," Opt. Lett. 38, 4138-4141 (2013)

[26] A. Agnesi, A. Greborio, F. Pirzio, G. Reali, and J. Aus, "40-fs $\mathrm{Yb} 3+$ :CaGdAlO4 laser pumped by a single- mode 350-mW laser diode," vol. IV, no. 9, pp. 2797-2799, 2012.

[27] E. Caracciolo, M. Kemnitzer, A. Guandalini, F. Pirzio, J. Aus der Au, and A. Agnesi, "28-W, $217 \mathrm{fs}$ solid-state $\mathrm{Yb}: \mathrm{CAlGdO}_{4}$ regenerative amplifiers," Opt. Lett. 38, 4131-4133 (2013)

[28] S. Ricaud, a Jaffres, K. Wentsch, a Suganuma, B. Viana, P. Loiseau, B. Weichelt, M. Abdou-Ahmed, a Voss, T. Graf, D. Rytz, C. Hönninger, E. Mottay, P. Georges, and F. Druon, "Femtosecond Yb:CaGdAlO4 thin-disk oscillator.," Optics letters, vol. 37, no. 19, pp. 3984-6, Oct. 2012.

[29] A. Greborio, A. Guandalini, and J. Aus der Au, "Sub-100 fs pulses with 12.5-W from Yb:CALGO based oscillators," Proc. SPIE 8235, Solid State Lasers XXI: Technololgy and Devices, 823511-823511 (2012).

[30] Y. Zaouter, J. Didierjean, F. Balembois, G. L. Leclin, F. Druon, P. Georges, J. Petit, P. Goldner, and B. Viana, "47-fs diode-pumped $\mathrm{Yb}<\sup >$ $3+</$ sup $>:$ CaGdAlO $<$ sub $>4</$ sub $>$ laser," Optics letters, vol. 31, no. 1 , pp. 119-121, 2006.

[31] Julien Pouysegur, Martin Delaigue, Yoann Zaouter, Clemens Hönninger, Eric Mottay, Anaël Jaffrès, Pascal Loiseau, Bruno Viana, Patrick Georges, and Frédéric Druon, "Sub-100-fs Yb:CALGO nonlinear regenerative amplifier," Opt. Lett. 38, 5180-5183 (2013) 


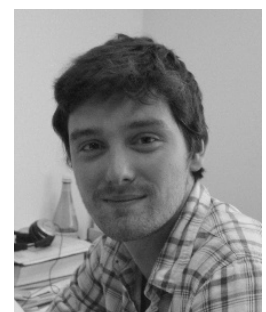

Julien Pouysegur was born in Bruges, France, in 1988. He received the B.S. and M.S. degrees in Laser, Matter and Nanosciences from the University of Bordeaux, in 2011.

He is currently pursuing the Ph.D. degree in Laser development between the Insitut d'Optique Graduate School (IOGS), Paris, France and the Company Amplitude Systèmes, Bordeaux, France.

His current research interests includes the development of High Power femtosecond amplifiers using Yb-doped crystals and the employment of nonlinear effects in order to decrease the pulse duration of the pulses.

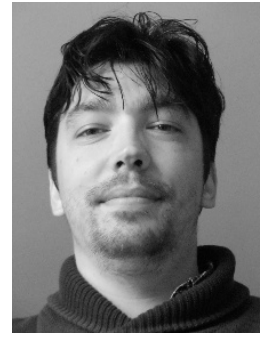

Martin Delaigue received an engineering degree in optics from Ecole Supérieure d'Optique, Orsay and a Master of Science degree in optics and photonics from Université Paris Sud, Orsay-France in 2003 and the Ph.D. degree in 2006 from Université Bordeaux 1-France. His Ph.D. work was focused on the development on novel architectures to increase the average power of femtosecond laser sources using Ti:Sapphire and Yb-doped crystals as active medium. In 2007, he joins the research and development department of Amplitude Systèmes and develops the new generation of crystal based amplifiers. His current research interests are in high power femtosecond laser sources based on crystalline architectures for industrial applications.

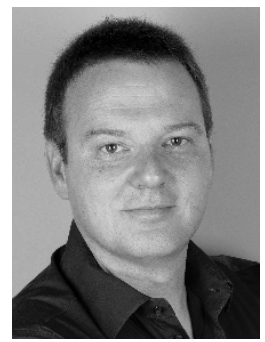

Clemens Hönninger studied Physics at the University of Heidelberg, Germany, where he graduated in 1994. During his $\mathrm{PhD}$ thesis at the Swiss Federal Institute of Technology in Zurich, from 1995 to 1998, he demonstrated the first femtosecond lasers based on Ytterbium-doped laser media. He continued these research activities during a postdoctoral stage at the University of Bordeaux, France, in 1999 and 2000. He joined Amplitude Systèmes in 2002. Prior to this he gained industrial experience as project leader at Trumpf Laser $\mathrm{GmbH}$, Germany and more recently as group leader at JT Optical Engine $\mathrm{GmbH}$ in Jena, Germany. $\mathrm{He}$ is VP Research and Development at Amplitude Systemes. His research activities are in industrial femtosecond lasers based on crystaline and fiber architectures.

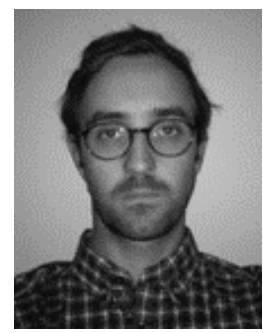

Yoann Zaouter was born in Alfortville, France, on May 18th, 1981. He received an engineering degree in optronics from IFIPS - Université Paris Sud, Orsay - France in 2004, a Master of Science degree in optics and photonics from Université Paris Sud, Orsay - France, in 2005 and a Ph.D. degree in Laser, Matter and Nanoscience from Université de Bordeaux, Bordeaux - France in 2008. His Ph.D work was carried out in collaboration with the company Amplitude Systemes, Pessac - France, and was focused on the development of novel ultrafast fiber laser architectures for industrial and scientific applications. In 2008, he joins the research and development department of Amplitude Systemes and developed the first generation of industrial ultrafast fiber lasers. Since 2009, he heads a joint research and development laboratory shared with the laser development team at Laboratoire Charly Fabry, Institut d'Optique, Palaiseau, France, where he develops laser concepts for average power and energy scaling of ultrafast laser technologies. His current research interests include ultrafast and nonlinear optics, fiber lasers and amplifiers, active and passive coherent combining of ultrafast amplifiers.

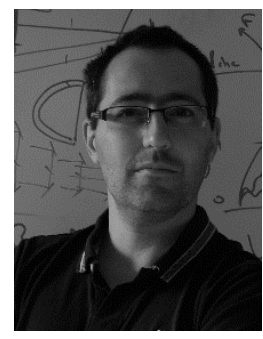

Frédéric Druon was born in Aubervilliers, France, in 1972. He received the engineer degree from the Electrical Engineering School of Paris (Sup élec), Gif-sur-Yvette, France, in 1996, and the Ph.D degree from the Institut d'Optique, Orsay, France, in 2000 , for work on ultracompact and efficient picosecond UV sources based on microchip lasers and on femtosecond diode-pumped laser systems.

He studied optics and photonics at the University of Paris XI. In 1997, he made his military service as a Research Visitor at the Center for Ultrafast Optical Science (CUOS), Ann Arbor, MI, where he worked on wave-front analysis and correction of terawatt laser chains. He was a Postdoctoral Researcher for one year and worked on mode-locking processes involving fast saturable absorbers. In 2001, he joined the Laboratoire Charles Fabry de l'Institut d'Optique, Centre National de la Recherche Scientifique (CNRS), Universit'e Paris Sud, Palaiseau, France. His current research interests consist in innovative ultrafast laser development. He received the "Fabry - De Gramont" Prize in 2005 and the "Jean Jerphagnon" Prize in 2011 for his research.

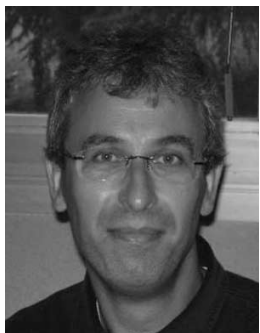

Patrick Georges was born in Metz, France, on September 13, 1962. He received the engineer degree from the Ecole Supérieure d'Optique, Palaiseau, France, in 1985, and the Ph.D. degree from the Institut d'Optique, Orsay, France, in 1989, for work on colliding pulses mode-locked dye lasers at different wavelengths and pulse compression.

$\mathrm{He}$ is currently a Senior Scientist at the Centre National de la Recherche Scientifique (CNRS), where he leads the Lasers Group, Laboratoire Charles Fabry at the Institut d'Optique, Palaiseau. His current research interests include diode-pumped solid-state lasers, new laser materials, picosecond and femtosecond lasers, high brightness laser diodes, fiber amplifier systems and optically pumped vertical surface emitting lasers. He is also engaged in the Apollon $10 \mathrm{PW}$ laser facility project and responsible of the Front-end.

Dr. Georges is a Fellow Member of the Optical Society and has served as Program Chair for the Cleo Europe 2013 conference.

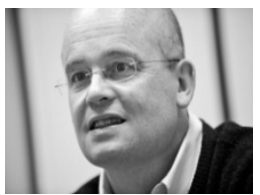

Eric Mottay is the president and CEO of Amplitude Systemes, France, a company he founded in 2001 and which is now a leader in diode-pumped ultrafast lasers. Eric graduated from the Ecole Superieure d'Optique, Orsay, in 1985, and has since specialized in laser development and manufacturing. 